\title{
Imported scrub typhus: first case in South America and review of the literature
}

\author{
Thomas Weitzel ${ }^{1,2^{*}}$ D, Mabel Aylwinn², Constanza Martínez-Valdebenito ${ }^{3}$, Ju Jiang ${ }^{4}$, Jose Manuel Munita ${ }^{2}$, \\ Luis Thompson ${ }^{2}$, Katia Abarca ${ }^{3}$ and Allen L. Richards ${ }^{4,5}$
}

\begin{abstract}
Background: Scrub typhus is a neglected vector-borne zoonosis causing life-threatening illnesses, endemic in the Asian-Pacific region and, as recently discovered, in southern Chile. Scrub typhus is rarely reported in travelers, most probably due to the lack of clinical experience and diagnostic tests in non-endemic countries. We report the first case of imported scrub typhus in South America.

Case presentation: A 62-year-old tourist from South Korea presented severely ill with fever, rash, and eschar in Santiago, Chile. Laboratory exams showed thrombocytopenia and elevated inflammation parameters, hepatic enzymes, and LDH. With the clinical suspicion of scrub typhus, empirical treatment with doxycycline was initiated and the patient recovered rapidly and without complications. The diagnosis was confirmed by IgM serology and by real-time PCR, which demonstrated infection with Orientia tsutsugamushi (Kawasaki clade).

Conclusions: Only due to the emerging clinical experience with endemic South American scrub typhus and the recent implementation of appropriate diagnostic techniques in Chile, were we able to firstly identify and adequately manage a severe case of imported scrub typhus in South America. Physicians attending febrile travelers need to be aware of this rickettsiosis, since it requires prompt treatment with doxycycline to avoid complications.
\end{abstract}

Keywords: Arthropod-borne diseases, Scrub typhus, Orientia tsutsugamushi, Travel, Imported infection

\section{Background}

Scrub typhus is a vector-borne zoonosis caused by Orientia species that manifests as an acute febrile disease and has a potentially severe outcome [1]. It is transmitted by the larval stage of trombiculid mites called 'chiggers'. After the bite of an infective chigger, a characteristic necrotic inoculation lesion termed eschar might develop, which typically contains high bacterial loads. The microorganism then spreads via lymphatics and blood, causing systemic manifestations and laboratory abnormalities such as elevated C-reactive protein (CRP) and liver enzymes [1]. Although widely under-recognized, scrub typhus is considered the most important rickettsial infection worldwide threatening over a billion people and causing more than a million cases per year

\footnotetext{
* Correspondence: thomas.weitzel@gmail.com

'Laboratorio Clínico, Clínica Alemana de Santiago, Facultad de Medicina

Clínica Alemana, Universidad del Desarrollo, Av. Vitacura, 5951 Santiago, Chile

${ }^{2}$ Servicio de Infectología, Clínica Alemana de Santiago, Facultad de Medicina

Clínica Alemana, Universidad del Desarrollo, Santiago, Chile

Full list of author information is available at the end of the article
}

with substantial mortality [2]. Until recently, scrub typhus was associated with a single species, O. tsutsugamushi, which exclusively occurred within the so-called 'Tsutsugamushi Triangle' ranging from Pakistan in the West, far-eastern Russia in the East to northern Australia in the South. However, recent reports of autochthonous cases in the Middle East and southern Chile have reshaped this epidemiological paradigm, suggesting a wider geographical distribution $[3,4]$.

Scrub typhus is very rarely diagnosed in travelers, with a total of $<40$ cases reported in the medical literature. Still, the problem might be under-recognized, since the initial clinical suspicion relies on the physicians' experience, routine laboratory tests are largely unavailable, and only few reference laboratories permit a definite diagnosis by molecular methods and/or culture. Here we report an Orientia tsutsugamushi infection in a traveler from South Korea visiting Chile, which was confirmed by molecular methods and serology.

(C) The Author(s). 2018 Open Access This article is distributed under the terms of the Creative Commons Attribution 4.0 International License (http://creativecommons.org/licenses/by/4.0/), which permits unrestricted use, distribution, and reproduction in any medium, provided you give appropriate credit to the original author(s) and the source, provide a link to the Creative Commons license, and indicate if changes were made. The Creative Commons Public Domain Dedication waiver (http://creativecommons.org/publicdomain/zero/1.0/) applies to the data made available in this article, unless otherwise stated. 


\section{Case presentation}

A 62-year-old South Korean tourist presented with fever, rash, and severe malaise two days after arrival in Chile. He reported flu-like symptoms with chills, headache, and myalgia, which had begun five days earlier. The patient was from Seoul, but had recently visited a farm south of Seoul. At presentation, the patient suffered intense headache and felt severely unwell. He was tachycardic $(108 \mathrm{bpm})$, had an axillar temperature of $38.8 \mathrm{C}$, and a generalized, non-pruritic maculopapular rash, more pronounced on the trunk and sparing palms, soles, and mucosa (Fig. 1, A and B). On his left posterior thigh, there was a painless necrotic lesion with a diameter of $6 \mathrm{~mm}$ and a surrounding red halo (Fig. 1, C). Complete blood count showed mild thrombocytopenia (91,000/ $\mu \mathrm{L})$, low hemoglobin $(13.0 \mathrm{~g} / \mathrm{dL})$, and normal leukocytes with a left shift (bands, 20\%), toxic granulation, and atypical (reactive) lymphocytes. Other laboratory exams revealed elevated inflammation parameters (ESR, $32 \mathrm{~mm} / \mathrm{h}$; CRP, $3.6 \mathrm{mg} / \mathrm{dL}$ ), slight hyponatremia $(134 \mathrm{mEq} / \mathrm{L})$, elevated hepatic enzymes (AST, $180 \mathrm{U} / \mathrm{L}$; ALT, $161 \mathrm{U} / \mathrm{L} ;$ GGT $327 \mathrm{U} / \mathrm{L} ; \mathrm{AP}, 208 \mathrm{U} / \mathrm{L})$ and LDH $(718 \mathrm{U} / \mathrm{L})$. A molecular respiratory panel $\left(\mathrm{xTAG}^{\circ} \mathrm{Re}-\right.$ spiratory Viral Panel FAST v2; Luminex, Austin, TX,

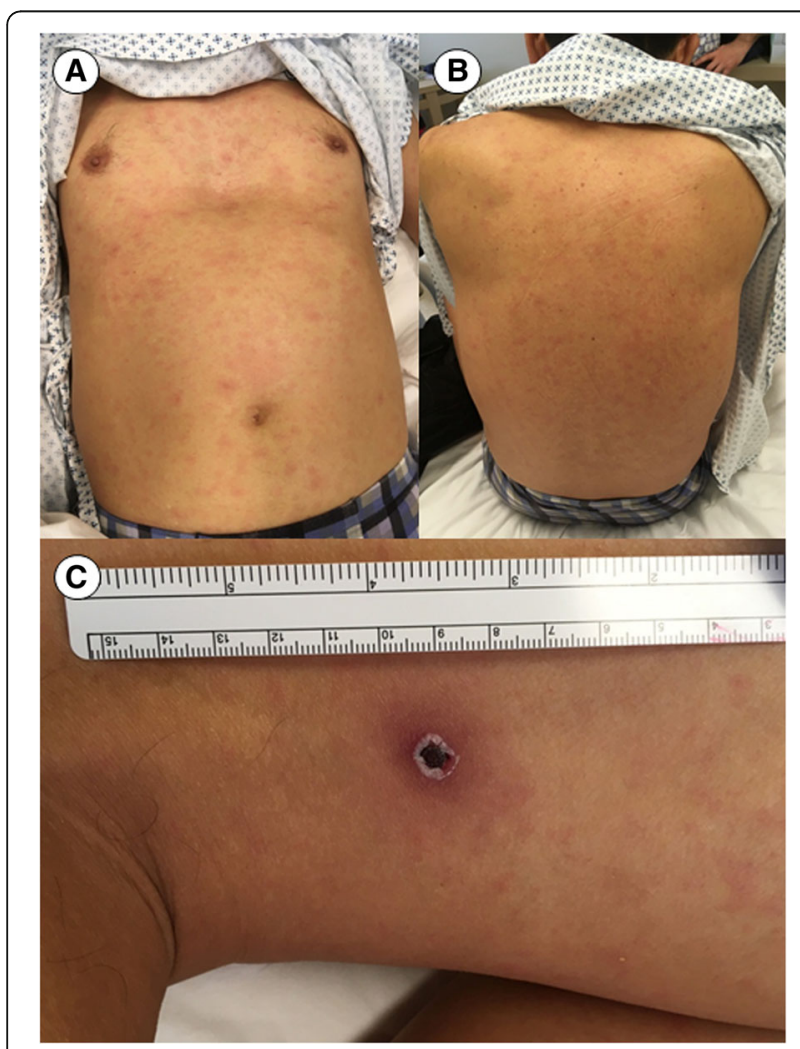

Fig. 1 Coarse maculopapular rash of South Korean patient, predominantly affecting the trunk $(\mathbf{a}, \mathbf{b})$, accompanied by characteristic necrotic eschar on the dorsal face of the left thigh (c)
USA) as well as serological assays (IgM) for EBV, CMV, and Parvovirus B19 were all negative.

Oral treatment with doxycycline $(100 \mathrm{mg}$ bid) was initiated in response to clinical suspicion of scrub typhus. Blood samples were drawn, the necrotic eschar was removed and placed in $70 \%$ alcohol, and a dry swab sample was taken from the base of the unroofed lesion. The following day, examination of serum by commercial ELISA (Scrub Typhus Detect, InBios International Inc., Seattle, WA, USA) was positive for IgM and negative for IgG antibodies. A commercial indirect IgG immunofluorescence test (Fuller Laboratories, Fullerton, CA, USA) utilizing acetone-fixed O. tsutsugamushi strains (Gilliam, Karp, Kato, Boryong) was positive at a dilution of 1:64 for the Karp antigen. DNA preparations from buffy coat as well as eschar material and swab samples were positive by quantitative real-time PCR (Otsu47) targeting the $O$. tsutsugamushi-specific $47 \mathrm{kD}$ protein gene (htrA) using primers and probe described previously [5]. From the eschar sample, rrs, htrA and $56 \mathrm{kDa}$ type specific antigen (tsa56) gene fragments were successfully amplified using semi-nested PCR assays (Table 1). Amplicons were purified and sequenced by 3500 Genetic Analyzer (Thermo Fisher Scientific, Waltham, MA, USA), the sequences from each primer were assembled with CodonCode Aligner (CodonCode Corporation, Centerville, MA, USA), and the consensus sequence of 1045,1456 and 1302 bp fragments were obtained for $r r s, h t r A$, and tsa56, respectively. Sequences were deposited in GenBank (accession numbers MG844362 [rrs], MG844360 [htrA], and MG844361 [tsa56]). Blast search (https://blast.ncbi.nlm.nih.gov/ Blast.cgi) revealed that the pathogen was closest to $O$. tsutsugamushi Kawasaki type strain, with 100\% identity for $\operatorname{rrs}$ (O. tsutsugamushi Kawasaki) and tsa56, including isolates from Korea (CBNU-2 and IIOC1217) [6] and Japan (Taguchi) [7]. Phylogenetic analysis also showed that the isolate clustered with Kawasaki type strains for both $r r s$ and tsa56, but was not related with the Orientia sp. Chiloe Island isolate recently discovered from Chile (Fig. 2).

With antibiotic treatment, fever subsided within $36 \mathrm{~h}$ and the patient was discharged after three days.

\section{Discussion}

Although scrub typhus represents an important public health issue in the Asia-Pacific region and is one of the most severe rickettsial infections, it is almost unrecognized in travelers and poorly covered in webbased information platforms and textbooks of Travel Medicine. A review from 2004 includes $\sim 20$ cases in travelers [8]; since then, $<15$ additional patients have been published, all as single case reports or small case series [9-19]. The GeoSentinel network reported only 
Table 1 Primers and probes used for diagnostic and phylogenetic analysis

\begin{tabular}{|c|c|c|c|c|}
\hline Primer ID & Sequence & Annealing temp. & PCR & Reference \\
\hline $16 s U 17 F$ & AGAGTTTGATCCTGGCTCAG & $56^{\circ} \mathrm{C}$ & First & [30] \\
\hline 16sOR1198R & TTTCCTATAGTTCCCGGCATT & $56^{\circ} \mathrm{C}$ & First \& second & \\
\hline $16 \mathrm{sO} 79 \mathrm{~F}$ & ATTAATGCTGAGCTTGCTTAGCAT & $56^{\circ} \mathrm{C}$ & Second & \\
\hline Otr47_263F & GTGCTAAGAAARGATGATACTTC & $54^{\circ} \mathrm{C}$ & First & [31] \\
\hline Otr1780R & AAATCGCCTTTAAACTAGATTTACTTATTA & $54^{\circ} \mathrm{C}$ & First \& second & \\
\hline Otr47F & TAAAGGTTAAGTTTATGAAAAAGGCATTT & $54{ }^{\circ} \mathrm{C}$ & Second & \\
\hline Otr56_498F & AATTAGTTTAGAATGGTTACCAC & $54{ }^{\circ} \mathrm{C}$ & First & \\
\hline r56_585F & AATGTCTGCGTTGTCGTTGC & $54{ }^{\circ} \mathrm{C}$ & Second & \\
\hline r56_2057 & TCCACATACACACCTTCAGC & $54{ }^{\circ} \mathrm{C}$ & First \& second & [32] \\
\hline OtsuFP630 & AACTGATTTITTCAAACTAATGCTGCT & $60^{\circ} \mathrm{C}$ & Real time & [5] \\
\hline OtsuRP747 & TATGCCTGAGTAAGATACRTGAATRGAATT & $60^{\circ} \mathrm{C}$ & Real time & [5] \\
\hline OtsuPR665 & 6FAM-TGGGTAGCTTTGGTGGACCGATGTTTAATCT-TAMRA & $60{ }^{\circ} \mathrm{C}$ & Real time & [5] \\
\hline
\end{tabular}

five confirmed cases among 47,915 ill travelers between 1996 and 2008 [20]. Most of these patients were diagnosed by serological tests of single (mostly convalescent) samples and only few were molecularly confirmed (mostly in endemic countries) $[18,19]$. In contrast, many experts postulate an increased risk of travel-associated scrub typhus due to the emergence of ecotourism (camping, trekking, rafting) in endemic areas [8, 21, 22], which is in accordance with experiences during military operations during World War II and the Vietnam and Korea conflicts, when scrub typhus affected thousands of soldiers [23]. The main reasons for the sustained paucity of reports in Travel Medicine, is most probably the lack of clinical experience and diagnostic tools in many non-endemic countries. In South America, for example, diagnostic tests for scrub typhus were unavailable until recently, and imported cases have never been described. In our case, the emerging clinical experience with endemic South American scrub typhus [3] and recent implementation of diagnostic techniques in Chile permitted the proper management and rapid diagnostic confirmation of this imported case.

Routinely, scrub typhus is diagnosed by serology, either by positive IgM or IgG seroconversion; though early cases are often seronegative. Definitive diagnosis mostly relies on molecular methods, preferably from eschar material, which stays positive even after initiation of treatment [24]. As in other severe rickettsial infections, empirical treatment should never be delayed due to diagnostic difficulties. In our case, PCR and serology

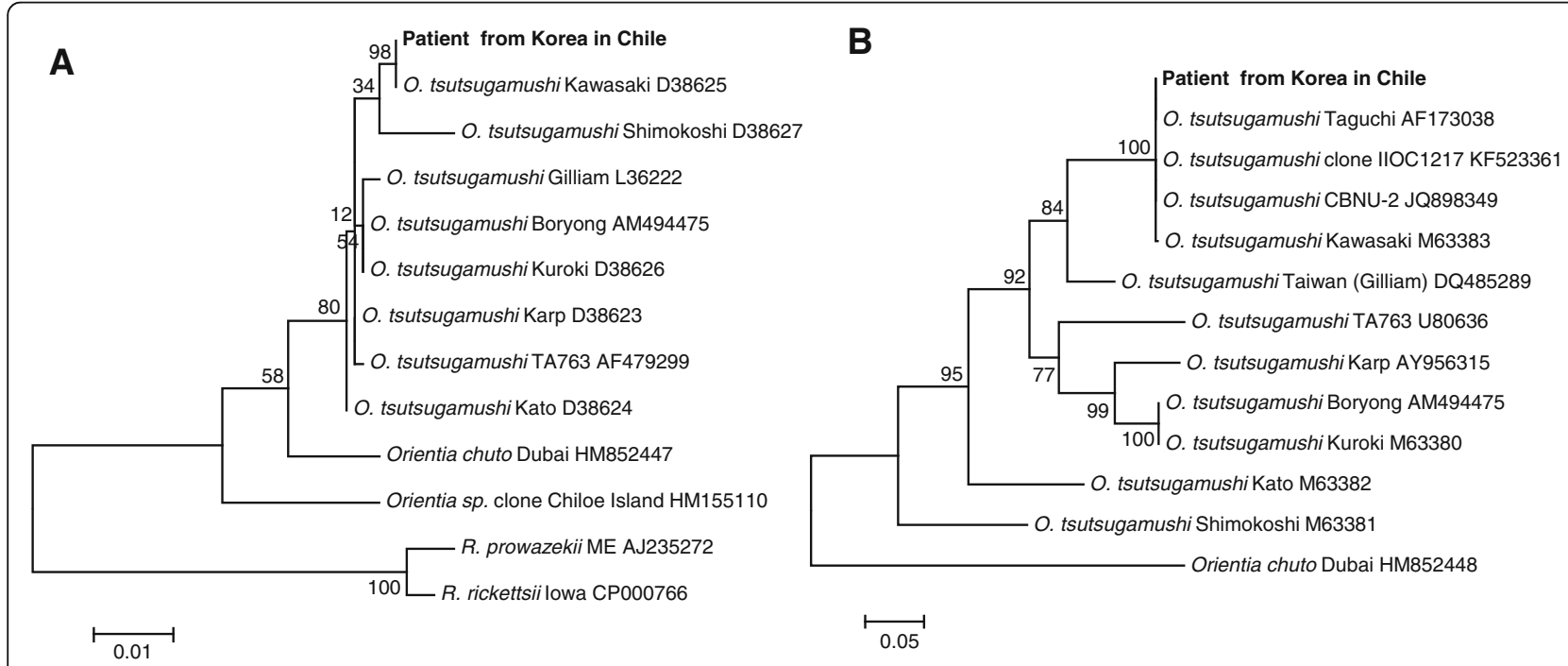

Fig. 2 Phylogenetic analysis of Orientia tsutsugamushi isolate amplified from eschar sample of patient from South Korea. The trees were constructed based on 970 bp rrs (a) and 593 bp tsa56 (b) gene fragments of the patient and O. tsutsugamushi type strains/isolates (GenBank accession numbers are shown next to each agent) using the Maximum Likelihood method with the Tamura-Nei model. Evolutionary analyses were conducted in MEGA7 and the values for the bootstrap test (1000 replicates) are shown next to the branches 
permitted a timely (but also retrospective) diagnosis, proving infection with $O$. tsutsugamushi Kawasaki strain, which was acquired in the Imsil district, a region of high scrub typhus incidence in South Korea [25].

Untreated scrub typhus has a high rate of complications and mortality, especially in naïve patients [21]. The immediate start of appropriate antibiotic treatment is therefore the main goal of clinical management and lowers the risk of severe manifestations. For this, the physicians' clinical experience and judgment is crucial. The most characteristic sign of scrub typhus is a necrotic skin lesion (eschar) at the inoculation site. It appears in $20-90 \%$ of patients, but seems to be more frequently present in naïve patients, e.g. travelers $[1,22]$. To detect the inconspicuous and painless lesion, a thorough physical exam is essential. If the eschar is not detected or patients do not present the lesion, diagnosis is often delayed and complications more probable. Scrub typhus may also affect domestic travelers. This phenomenon has been recognized in Asian countries such as Taiwan [26], and is also an emerging problem in Chilean travelers returning to the central metropolitan region from trips to endemic regions in southern Chile (unpublished data). The main differential diagnoses in eschar-positive febrile travelers are spotted fever group rickettsioses. In our patient, those included Japanese spotted fever caused by Rickettsia japonica and other endemic rickettsiae endemic in South Korea such as $R$. monacensis, R. felis, and R. akari [27, 28]. The centrifugal distribution of the rash, sparing palms and soles, was suggestive for scrub typhus, since rickettsial spotted fevers have a centripetal rash including palms and soles [29]. In comparison to the rash of patients with dengue or other arboviral infections, the rash in scrub typhus has a coarser and more irregular appearance. The presence of atypical lymphocytes might lead to the misdiagnosis of infective mononucleosis, especially in the presence of lymphadenopathy.

\section{Conclusions}

This first case of imported scrub typhus in South America highlights the need of physicians attending febrile travelers to be aware of this severe rickettsiosis. This includes knowledge of the endemic regions in Asia, its emergence in South America, and the recognition of the typical clinical manifestations. As in other rickettsial infections, rapid clinical recognition and prompt empirical treatment with doxycycline are crucial for a favorable patient's prognosis. Diagnostic tools, ideally molecular methods, are useful for retrospective confirmation, but are only available in specialized laboratories. Pre-travel consultations should include information on the risk and prevention of this infection, especially for travelers with outdoor activities such as camping and trekking in endemic regions.
Acknowledgments

We wish to thank Lorena Porte for her thoughtful manuscript input.

Funding

This work was partially supported by the Fondo Nacional de Desarrollo Científico y Tecnológico (FONDECYT N 1170810) and the Global Emerging Infectious Diseases Section, work unit \# A1402.

\section{Availability of data and materials}

All data are presented herein.

\section{Disclaimer}

The opinions and assertions contained in this paper are the private views of the authors and are not to be construed as official or as reflecting the views of the Department of the Navy, the Department of Defense nor the United States Government

A. L. Richards is an employee of the United States Government. This work was prepared as a part of his official duties. Title 17 U.S.C. \$105 provides that 'Copyright protection under this title is not available for any work of the United States Government'. Title 17 U.S.C. §101 defines a U.S. Government work as a work prepared by a military service member or employee of the U.S. Government as part of that person's official duties.

\section{Authors' contributions}

TW and ALR conceived the report. TW, CM-V, JJ, and ALR drafted the manuscript and contributed to the literature review. TW, MA, JMM, and LT attended the patient. All authors participated in the interpretation of relevant results, provided critical edits, and approved the final manuscript.

\section{Ethics approval and consent to participate}

Ethics approval for this case report was not sought as there was no human subjects study in which to participate. The patient consented that his personal and clinical information was used for scientific and teaching reasons

\section{Consent for publication}

The patient consented that information regarding his case was presented in this manuscript.

\section{Competing interests}

The authors declare that they have no competing interests.

\section{Publisher's Note}

Springer Nature remains neutral with regard to jurisdictional claims in published maps and institutional affiliations.

\section{Author details}

'Laboratorio Clínico, Clínica Alemana de Santiago, Facultad de Medicina Clínica Alemana, Universidad del Desarrollo, Av. Vitacura, 5951 Santiago, Chile. ${ }^{2}$ Servicio de Infectología, Clínica Alemana de Santiago, Facultad de Medicina Clínica Alemana, Universidad del Desarrollo, Santiago, Chile. ${ }^{3}$ Departamento de Enfermedades Infecciosas e Inmunología Pediátricas, Escuela de Medicina, Pontificia Universidad Católica de Chile, Santiago, Chile. ${ }^{4}$ Viral and Rickettsial Diseases Department, Naval Medical Research Center, Silver Spring, MD, USA. ${ }^{5}$ Department of Preventive Medicine and Biostatistics, Uniformed Services University of the Health Sciences, Bethesda, MD, USA.

Received: 5 July 2018 Accepted: 8 August 2018

Published online: 16 August 2018

References

1. Kim IS, Walker DH. Scrub typhus. In: Guerrant RL, Walker DH, Weller PF, editors. Tropical infectious diseases: principles, pathogens and practice. 3rd ed. New York: Elsevier; 2011. p. 334-8.

2. Paris DH, Shelite TR, Day NP, Walker DH. Unresolved problems related to scrub typhus: a seriously neglected life-threatening disease. Am J Trop Med Hyg. 2013;89:301-7.

3. Weitzel T, Dittrich S, López J, Phuklia W, Martinez-Valdebenito C, Velásquez K, Blacksell SD, Paris DH, Abarca K. Endemic scrub typhus in South America. N Engl J Med. 2016;375:954-61. 
4. Jiang J, Richards AL. Scrub typhus: no longer restricted to the Tsutsugamushi Triangle. Trop Med Infect Dis. 2018;3:11.

5. Jiang J, Chan TC, Temenak JJ, Dasch GA, Ching W-M, Richards AL. Development of a quantitative real-time PCR assay specific for Orientia tsutsugamushi. Am J Trop Med Hyg. 2004;70:351-6.

6. Jeong HW, Choi YK, Baek YH, Seong MH. Phylogenetic analysis of the 56kDa type-specific protein genes of Orientia tsutsugamushi in Central Korea. J Korean Med Sci. 2012;27:1315-9.

7. Enatsu T, Urakami H, Tamura A. Phylogenetic analysis of Orientia tsutsugamushi strains based on the sequence homologies of 56-kDa typespecific antigen genes. FEMS Microbiol Lett. 1999;180:163-9.

8. Jensenius M, Fournier PE, Raoult D. Rickettsioses and the international traveler. Clin Infect Dis. 2004:39:1493-9.

9. Seilmaier M, Guggemos W, Böhme CC, Löscher T. Tsutsugamushi fever after travel to Southeast Asia. Dtsch Med Wochenschr. 2004;129:2233-5.

10. Jensenius M, Montelius R, Berild D, Vene S. Scrub typhus imported to Scandinavia. Scand J Infect Dis. 2006:38:200-2.

11. Nachega JB, Bottieau E, Zech F, Van Gompel A. Travel-acquired scrub typhus: emphasis on the differential diagnosis, treatment, and prevention strategies. J Travel Med. 2007;14:352-5.

12. Izzard L, Fuller A, Blacksell SD, Paris DH, Richards AL, Aukkanit N, Nguyen C, Jiang J, Fenwick S, Day NP, et al. Isolation of a novel Orientia species (O. chuto sp. nov.) from a patient infected in Dubai. J Clin Microbiol. 2010;48: 4404-9.

13. Keller C, Fähndrich K, Müller W, Nüsslein TG, Fleischer B, Hegasy G. Epistaxis, maculopapular rash and fever in a German boy after a stay in Thailand caused by Orientia tsutsugamushi. Klin Padiatr. 2012;224:270-1.

14. Edouard S, Subramanian G, Lefevre B, Dos Santos A, Pouedras P, Poinsignon Y, Mediannikov O, Raoult D. Co-infection with Arsenophonus nasoniae and Orientia tsutsugamushi in a traveler. Vector Borne Zoonotic Dis. 2013;13:565-71.

15. Vliegenthart-Jongbloed K, de Mendonça MM, Slobbe L, Beersma MF, van Genderen PJ. Imported scrub typhus in The Netherlands. Travel Med Infect Dis. 2013;11:197-9.

16. Henry J, Beckwith H, Dubrey S. Scrub typhus after a trip to India. QJM. 2014; 107:483

17. Carémil F, Wey PF, Nourry É, Jean FX, Dardare É, Gérome P, de Charry C, Alberti N, Lamblin A. Septic shock with multiple organ failure after returning from Southeast Asia. Med Sante Trop. 2016;26:31-4.

18. Suzuki Y, Shimanuki M, Seto J, Yahagi K, Mizuta K. A case of scrub typhus imported from South Korea to Yamagata, Japan. Jpn J Infect Dis. 2016;69:454-6.

19. Imad HA, Tanyaratsrisakul S, Piyaphanee W, Wattanagoon Y. Skin lesion from Maldives: classic but forgotten. Travel Med Infect Dis. 2017;17:74-5.

20. Jensenius M, Davis X, von Sonnenburg F, Schwartz E, Keystone JS, Leder K, Lopéz-Véléz R, Caumes E, Cramer JP, Chen L, et al. Multicenter GeoSentinel analysis of rickettsial diseases in international travelers, 1996-2008. Emerg Infect Dis. 2009:15:1791-8.

21. Watt G, Kantipong P. Orientia tsutsugamushi and scrub typhus. In: Raoult D, Parola P, editors. Rickettsial diseases. New York: Informa Healthcare; 2007. p. 237-56

22. Aung AK, Spelman DW, Murray RJ, Graves S. Rickettsial infections in Southeast Asia: implications for local populace and febrile returned travelers. Am J Trop Med Hyg. 2014;91:451-60.

23. Kelly DJ, Richards AL, Temenak J, Strickman D, Dasch GA. The past and present threat of rickettsial diseases to military medicine and international public health. Clin Infect Dis. 2002;34(Suppl 4):S145-69.

24. Koh GC, Maude RJ, Paris DH, Newton PN, Blacksell SD. Diagnosis of scrub typhus. Am J Trop Med Hyg. 2010;82:368-70.

25. Jin HS, Chu C, Han DY. Spatial distribution analysis of scrub typhus in Korea. Osong Public Health Res Perspect. 2013;4:4-15.

26. Wang YC, Chen PC, Lee KF, Wu YC, Chiu CH. Scrub typhus cases in a teaching hospital in Penghu, Taiwan, 2006-2010. Vector Borne Zoonotic Dis. 2013;13:154-9.

27. Choi YJ, Jang WJ, Ryu JS, Lee SH, Park KH, Paik HS, Koh YS, Choi MS, Kim IS. Spotted fever group and typhus group rickettsioses in humans, South Korea. Emerg Infect Dis. 2005;11:237-44

28. Kim YS, Choi YJ, Lee KM, Ahn KJ, Kim HC, Klein T, Jiang J, Richards A, Park $\mathrm{KH}$, Jang WJ. First isolation of Rickettsia monacensis from a patient in South Korea. Microbiol Immunol. 2017;61:258-63.

29. Mahara F. Rickettsioses in Japan and the far east. Ann N Y Acad Sci. 2006; 1078:60-73.
30. Srivastava S, Singh V, Kumar V, Verma PC, Srivastava R, Basu V, Gupta V, Rawat AK. Identification of regulatory elements in $16 \mathrm{~S}$ rRNA gene of Acinetobacter species isolated from water sample. Bioinformation. 2008;3:173-6.

31. Jiang J, Paris DH, Blacksell SD, Aukkanit N, Newton PN, Phetsouvanh B, Izzard L, Stenos J, Graves SR, Day NP, et al. Diversity of the $47 \mathrm{kDa}$ HtrA translated amino acid sequence among human isolates of Orientia. Vector Borne Zoonotic Dis. 2013;13:367-75.

32. Qiang Y, Tamura A, Urakami H, Makisaka Y, Koyama S, Fukuhara M, Kadosaka T. Phylogenetic characterization of Orientia tsutsugamushi isolated in Taiwan according to the sequence homologies of 56-kDa type-specific antigen genes. Microbiol Immunol. 2003;47:577-83.

\section{Ready to submit your research? Choose BMC and benefit from:}

- fast, convenient online submission

- thorough peer review by experienced researchers in your field

- rapid publication on acceptance

- support for research data, including large and complex data types

- gold Open Access which fosters wider collaboration and increased citations

- maximum visibility for your research: over $100 \mathrm{M}$ website views per year

At $\mathrm{BMC}$, research is always in progress.

Learn more biomedcentral.com/submissions 